INTERVIEW

\section{Synthetische Biologie: Zwischen Fiktion und Realität}

Interview mit Dr. Markus Schmidt über das BIO-FICTION Science Art FilmFestival (23.-25. Oktober 2014, Wien, Österreich)

Synthetische Biologie ist ein aufstrebendes Forschungsgebiet, das Wissen, Ansätze und Methoden der Biotechnologie, Verfahrenstechnik, Informatik und verwandte Disziplinen umfasst. Das Hauptziel ist es, Organismen mit neuen Eigenschaften, die sonst in der Natur nicht existieren, zu schaffen. Zukünftige Anwendungen der Synthetischen Biologie werden möglicherweise einen wichtigen Beitrag zur Bioökonomie und im medizinischen Bereich leisten und Lösungen für Umweltprobleme anbieten können. SYNENERGENE ist ein von der EU gefördertes Projekt zu gesellschaftlichen Auswirkungen dieser neuen Technologie. In diesem TATuP-Interview sprechen wir mit Markus Schmidt, Gründer der Biofaction KG, einem Forschungsunternehmen in Wien, das sich mit Technikfolgenabschätzung zu neuen Biotechnologien sowie mit Wissenschaftskommunikation und Kunst-Wissenschaft-Kooperationen beschäftigt. Im Oktober 2014 haben er und sein Team im Rahmen des SYNENERGENEProjekts das zweite „BIO-FICTION Science, Art \& Film"-Festival organisiert, das ethische, rechtliche und ökonomische Aspekte sowie langfristige gesellschaftliche Auswirkungen der Synthetischen Biologie nicht nur in zahlreichen Vorträgen und Sessions, sondern insbesondere auch durch verschiedene Filme und Kunstinstallationen betrachtet hat. Das Interview für TATuP führte Antonina Khodzhaeva, European Network Science Centers \& Museums (ECSITE), Brüssel.

Antonina Khodzhaeva (TATuP): Ende September 2014 haben die Europäische Kommission und ihre wissenschaftlichen Beratungsgremien im Bereich Verbraucherschutz, Gesundheit und Umwelt für „Verbrauchersicherheit“ (Scientific Committee on Consumer Safety, SCCS),
„Gesundheits- und Umweltrisiken“ (Scientific Committee on Health and Environmental Risks, SCHER) und „Neu auftretende und neu identifizierte Gesundheitsrisiken" (Scientific Committee on Emerging and Newly Identified Health Risks, SCENIHR) ein Gutachten zur Definition der Synthetischen Biologie veröffentlicht. ${ }^{1} \mathrm{Sie}$ haben als externer Experte an diesem Gutachten und an anderen einschlägigen Aktivitäten dieser Ausschüsse mitgearbeitet. Was ist Synthetische Biologie? Wie definiert man sie?

Markus Schmidt (MS): Im Beratungsgremium haben wir uns auf folgende Definition geeinigt:

„SynBio is the application of science, technology and engineering to facilitate and accelerate the design, manufacture and/or modification of genetic materials in living organisms."

Interessant an der Definition ist, dass sie relativ breit angelegt ist und wenige technische Details voraussetzt. Im Prinzip wird ganz allgemein die Veränderung von biologischer Materie als Synthetische Biologie bezeichnet. Wir hatten uns zunächst überlegt, ob es einen Kriterienkatalog geben könnte, anhand dessen festgestellt werden kann, wann es sich um Synthetische Biologie handelt. Nach langer Diskussion sind wir aber zu der Ansicht gekommen, dass das nicht funktionieren kann, weil man zwar bestimmte Kriterien wie etwa Standardisierung, Modularisierung etc. heranziehen könnte, jedoch keine klare, nicht willkürliche Grenze etwa zwischen wenig standardisierten zu wirklich standardisierten Technologien gezogen werden kann. Anders formuliert: $\mathrm{Ab}$ wie vielen synthetischen Basen ist ein Organismus synthetisch? In dem Gutachten werden sechs Teilbereiche der Synthetischen Biologie vorgestellt: DNA-Synthese, Minimalgenom, Protozellen, Xenobiologie, Metabolic Engineering im weitesten Sinne sowie alles, was mit AmateurBiologie und Do-It-Yourself-Biologie zu tun hat.

TATuP: Es wurden in diesem Zusammenhang weitere Gutachten für die EU-Kommission erstellt. Welche Themen wurden da behandelt?

MS: Im zweiten Gutachten ${ }^{2}$ ging es um Risikofragen. Wird die Synthetische Biologie etwa in den nächsten zehn Jahren neue Risiken hervor- 
bringen? Werden die bestehenden Risikobewertungsmethoden ausreichen? Das dritte und letzte Gutachten $^{3}$ behandelt Fragen zur Biodiversität und wie man ein Forschungsprogramm ausgestalten könnte, das sich schwerpunktmäßig mit Sicherheitsfragen beschäftigt und die Risikobewertungsmethoden verbessert.

TATuP: Wie entstand die Idee, das Filmfestival zu organisieren?

MS: Wir haben schon im Jahr 2009 einen Dokumentarfilm zur Synthetischen Biologie im Rahmen des ebenfalls von der EU geförderten Projekts SynBioSafe gemacht ${ }^{4}$ und dabei realisiert, dass man dieses Thema auf viele verschiedene Arten behandeln kann. Mit nur einem Film konnten wir nur eine Geschichte erzählen, und wir wollten noch mehr Geschichten hören und noch mehr Filme sehen. Das war die Ursprungsidee und aus der entstand das erste Bio·Fiction-Festival im Jahr 2011. Wir haben Leute eingeladen, ihre Perspektiven über Synthetische Biologie darzustellen, und es haben sich, zum Teil miteinander arbeitend, sowohl Künstler und Designer als auch Natur- und Sozialwissenschaftler und andere beteiligt. Das Medium des Films macht es möglich, die Hoffnungen und Gefahren in einer Weise darzustellen, die im akademischen Diskurs eher mit hochgezogenen Augenbrauen wahrgenommen würden. Der Film kann mit spekulativer Ethik und kreativem Design anders agieren als die Wissenschaft. In solch einem experimentellen Umfeld hat man auch die Freiheit, über langfristige Entwicklungsmöglichkeiten einer Technologie zu reflektieren.

TATuP: Das erste Bio·Fiction-Festival fand im Jahr 2011 statt. Wie hat sich das Festival seitdem geändert? Was waren die Erwartungen an das Festival 2014?

MS: Beim ersten Festival wurden Filme gezeigt, Vorträge gehalten und es gab eine Kunstausstellung. Diesmal war es im Prinzip ähnlich. Aber wir hatten im Oktober 2014 mehr Filme, die wirklich gut zum Thema passten. Beim zweiten Mal war das Festival schon bekannter, man wusste schon, was von dem Festival zu erwarten ist. Wir hatten beim zweiten Festival neben den Filmen, Vorträgen und Diskussionen zudem eine Ausstellung von Kunstobjekten und eine Kunstperformance im „Narrenturm“ des Naturhistorischen Museum Wiens im Programm. Das Festival wurde dieses Mal an verschiedenen Orten in Wien durchgeführt. Dabei fanden auch zahlreiche Live-Demonstrationen und KunstPerformances statt. Am letzten Tag hatten wir zudem noch Workshops zum Thema Biohacking/ Do-it-yourself-Biology und Unternehmertum. Wir haben im Rahmen der Aktivitäten des SYNENERGENE-Projekts zur Do-it-yourself-Biologie fünf Biohacker-Gruppen eingeladen, die auch ihre Objekte bzw. Prototypen mitgebracht hatten. Während der Live-Demonstrationen konnte man die Prototypen (z. B. einen Bakterienfilm-3D-Drucker, eine bakterielle Biotinte, ein Minilabor in Koffergröße oder einen Hefe-Fotoapparat) näher anschauen oder sie ausprobieren. Das brachte ein interaktives Element hinein. Die Hoffnung hinsichtlich des gesamten Festivals war, dass ein lebendiger Austausch innerhalb dieser sehr bunten Truppe von Leuten zustande kommt, zwischen Künstlern, Natur-, Sozial- und Geisteswissenschaftlern, Technikfolgenabschätzern, Ingenieuren, Journalisten, Filmemachern, Do-It-Yourself-Biologen und anderen. Diese Erwartung hat sich erfüllt.

TATuP: Wie profitiert die Technikfolgenabschätzung der Synthetischen Biologie von der Einbeziehung von Kunst und Filmen?

MS: Es gibt in der Wissenschaft, Industrie und Politik ein großes Interesse an der Synthetischen Biologie. Es werden Rahmenbedingungen geschaffen, Projekte und Programme gefördert, Exzellenzzentren gegründet. Forschungsinstitute, Start-ups oder Industrieunternehmen entwickeln neue Produkte, man investiert in Forschung und Entwicklung. Das Feld entwickelt sich zügig. Und dem folgt zumeist die Technikfolgenabschätzung, die das bewertet, möglichst frühzeitig, und die Entwicklungen der Wissenschaft und Technologien vornimmt. Bei den Methoden, die die Technikfolgenabschätzung anwendet, kommt noch sehr viel aus der Soziologie, und sie ist diesbezüglich limitiert in der Art und Weise, wie sie Fragen stellt. Über die Einbeziehung der Kunst und des Films im Speziellen kann man 
den Diskussions- und Reflexionsraum erweitern. Durch die Einbindung der Kunst entsteht eine Möglichkeit, komplexe Themenstellungen sehr prägnant auf den Punkt zu bringen, und auch zur Wahrnehmung von Perspektiven einzuladen, die im akademischen und auch im TA-Umfeld so nicht oder nur sehr selten vorkommen.

TATuP: Die Filme und die Fiktion bieten mehr Raum für Spekulation. Aber wie können fiktionale Darstellungen dazu beitragen, über die ethischen und gesellschaftlichen Implikationen der Synthetischen Biologie zu reflektieren, besonders im Vergleich zu anderen Methoden der Technikfolgenabschätzung? Könnten Sie das vielleicht an einem Beispiel erläutern?

MS: In den Filmen, in denen es ein fiktives Element gibt, kann man Geschichten erzählen, bei denen man sich nicht streng an die „Fakten“ halten muss. Damit lassen sich - in eine Geschichte verpackt - Befürchtungen und Hoffnungen besser ausdrücken. „Bruce“ (2011), der Siegerfilm des ersten Festivals in der ZeichentrickfilmKategorie, ${ }^{5}$ greift z. B. ein zentrales Element des Diskurses zur Synthetischen Biologie auf: die Annahme, dass in Zukunft mit biotechnischen Mitteln Leben erschaffen werden kann. In diesem Film jagt ein Mensch, wohl zu Hause, ein kleines, von ihm mittels Joystick kontrolliertes Männlein über einen Parcours auf Wandregalen, wie in einem Computerspiel. Das erzeugt durchaus auch Heiterkeit beim Filmpublikum. Dem schwer atmenden Männlein misslingt dann aber ein Sprung, und es verletzt sich schwer durch einen Sturz auf den Boden. Obwohl es noch lebt, „entsorgt" es der Spieler, der es selbst belebt hatte, in einem Plastikbeutel, in dem das Männlein dann erstickt. Es wird also auf die bedeutende aktuelle Kulturpraxis „Gaming“ Bezug genommen und mit dem unheimlichen Gefühl, dass die Vision Künstliches Lebens erzeugen kann, auf eine Weise gespielt, die zum Hinterfragen von zukunftsbezogenen Hoffnungen und Ängsten anregt. Kommunikation ist sehr stark narrativ geprägt. Man kann in den Geschichten die Dinge ansprechen, die man sich in einem rein faktenbasierten Diskurs, nicht zu thematisieren trauen würde. Und wenn jemand von außerhalb des akademischen Diskurses in diesem mitreden will, besteht immer die Gefahr, dass er nicht ernst genommen wird, weil er die Spielregeln, den Code, das Vokabular einer akademischen Diskussion womöglich nicht beherrscht. Das ist bei Veranstaltungen wie BIO-FICTION anders. Ich glaube sogar, dass man vielschichtige komplexe Zusammenhänge mit Bildern oft besser erklären kann als mit den üblichen diskursiven Mitteln. Es sind aber nicht nur die Mittel der Fiktion, die dies in unserem Festival befördern, sondern es ist insbesondere auch das Medium Film, das dies ermöglicht. Es geht weniger um die Technologie im engeren Sinne, sondern eher um die Hoffnungen, die man in sie setzt, z. B. hinsichtlich der Krankheiten, die geheilt werden sollen, um die Visionen, die in der Entwicklung des Felds artikuliert werden, und die unterschiedlichen ökonomischen Rahmenbedingungen. Die Technologie selbst ist weniger Gegenstand des künstlerischen Ausdrucks, sondern es geht eher um das Prinzip dahinter und die Anwendungen der Technologie.

TATuP: Welche Rolle spielt denn die Fiktion im Zusammenhang des gesellschaftlichen Umgangs mit Technologie? Reflektiert Fiktion die Gesellschaft und ihre Interaktion mit der Technologie oder werden die Gesellschaft und ihre Interaktionen mit der Technologie durch die Fiktion geprägt?

MS: Beides. Die Fiktion prägt sicher. Wenn man einen neuen Kinofilm sieht, dann weiß man natürlich, dass das keine echte Erfahrung ist, sondern Fiktion, aber im Hinterkopf kann so ein Referenzpunkt verankert werden, der sicherlich auch Entscheidungen beeinflusst, ähnlich unbewusst wie Werbung funktioniert. Umgekehrt kann die Fiktion natürlich auch Interaktion zwischen Gesellschaft und Technik sowie verbreitete Zukunftsvisionen reflektieren. Ich sehe hier kein Entweder-oder oder einen Widerspruch. Es gibt allerdings auch Unterschiede zwischen künstlerischen Filmen und ganz oder überwiegend auf bloße Unterhaltung abzielenden Hollywoodfilme. Die Geschichten, die in solchen HollywoodFilmen erzählt werden, sind i. d. R. stereotypisch, in 4 von 5 Fällen mit einem Happy End gesegnet. Experimentelle Formate sind hingegen nicht auf ein Massenpublikum hin optimiert. 
TATuP: Wie kann ein solches künstlerisches Festivalformat zur Governance von neuen, emergierenden Technologien beitragen, besonders in Bezug auf die öffentliche Partizipation?

MS: Für die breitere Bevölkerung ist es gar nicht so einfach, in einem akademischen Diskurs über Technologieentwicklung mitzudiskutieren. Aber genau dann, wenn man, wie auch im SYNENERGENE-Projekt, verschiedene Stakeholder einbeziehen möchte, trägt ein solches Festival zu einer breiteren Öffnung bei, wenn auch Designer, Künstler und Filmmacher einen wenig limitierten Zugang zur Darstellung und Entwicklung von $\mathrm{Zu}-$ kunftsvisionen einer neuen Technologie schaffen. Sie stellen mehr Möglichkeiten zur Verfügung, um zu zeigen und zu reflektieren, wie eine Technologie im Zusammenspiel mit der gesellschaftlichen Entwicklung aussehen könnte. Was die Entwicklung einer Technologie betrifft, ist es ja nicht so, dass man nur dagegen oder dafür sein muss. Seitens der Politik besteht z. B. ein Interesse, Sicherheitsstandards zu setzen. Man muss nicht einfach für oder gegen diese Standards sein, sondern es gibt vielschichtigere Entwicklungspfade und da hilft sicherlich das Einbeziehen von Künstlern und Designern, neue Entwicklungsperspektiven zu eröffnen. Die Biohacker bzw. DIY-Biologen sind auch ein Beispiel einer besonderen Gruppe. Sie sind in ihrer Tätigkeit nicht unbedingt vom Gewinnstreben getrieben, im Gegensatz zur Industrie, die immer gewinnorientiert agiert. Die Europäische Kommission ist auch daran interessiert, dass eine wissensbasierte Biookönomie Realität wird, das heißt, dass die Investitionen im Sektor Früchte tragen und Produkte auf den Markt kommen sollen, die zum Wirtschaftswachstum beitragen, Menschen beschäftigt werden und so weiter. In diesem Zusammenhang spielt der ökonomische Fokus eine sehr große Rolle, aber es gibt auch andere Perspektiven, die berücksichtigt werden müssen. Eine Gruppe von Biohackern hat ein Diagnostikmodul gebaut, das in Entwicklungsländern verwendet werden könnte. Man kann damit nicht viel Geld verdienen, aber es hilft den Leuten in der „Dritten Welt", Malaria zu diagnostizieren. Einer rein ökonomischen Logik folgend, würde man das nicht machen. Aber es gibt andere Zielsetzungen, die sich nicht unbedingt der Ökonomie subsum- mieren lassen und trotzdem eine Existenzberechtigung haben. Das Festival trägt dazu bei, eine drohende Eindimensionalität der Entwicklungsoptionen von vorne herein auszuschließen. Das war im Übrigen auch der Grund für die Benennung der Konferenzsessions in: Homo oeconomicus, Homo societatis, Homo culturalis etc.

TATuP: Auf dem Festival wurden auch einige Projekte vorgestellt, in denen Künstler und Wissenschaftler zusammengearbeitet haben. Welche Bedeutung kann die Kooperation zwischen Wissenschaftlern und Künstlern insbesondere für das Verständnis möglicher gesellschaftlicher Folgen in der weiteren Entwicklung der Synthetischen Biologie haben?

MS: Generell denke ich, dass solche Kooperationen Freiheit für beide Seiten, sowohl für Forscher, als auch für Künstler, ermöglichen. Wir hatten bei der letzten Ausstellung ein Team aus Künstlern und Wissenschaftlern, in deren Projekt es um den Ursprung des Lebens und um die Biochemie der Ursuppe ging. Im Laufe der Ausstellung haben sie eine modifizierte Version des Miller-Urey-Experiments aus den 1950er Jahren durchgeführt - statt der Ursuppe wurde steriles Meerwasser und sterile Luft aus dem Jahr 2011 verwendet. Da hieß es von einigen Wissenschaftlern, dass es nutzlos und eine Geldverschwendung sei, dieses modifizierte Experiment durchzuführen, weil etwa der Sauerstoffgehalt in der heutigen Luft das Ursuppenexperiment behindern würde. In diesem Fall hatten dann Wissenschaftler über die Kunst die wissenschaftliche Freiheit zurückbekommen, dieses Experiment durchzuführen, was in der Peer-Gruppe in der Biochemie nicht machbar gewesen wäre. Während der Ausstellung wurden von dem LiveExperiment Proben genommen und in der Analyse tatsächlich neue Ursuppenmoleküle gefunden, entgegen den Annahmen der Biochemie Experten.

TATuP: Das Festival wurde ja als Teil des SYNENERGENE-Projektes organisiert. Wie trägt das Festival zu Responsible Research and Innovation (RRI) (Verantwortungsvoller Forschung und Innovation) im Bereich der Synthetischen Biologie bei? 
MS: Ich denke RRI, so wie ich dieses Konzept verstehe, bedeutet, dass man durch die Einbeziehung von unterschiedlichen Interessengruppen viele Perspektiven und Stimmen hören möchte und dass die Entscheidungen nicht ausschließlich nach ökonomischen Interessen getroffen werden. Im Projekt SYNENERGENE geht es in unserem Arbeitspaket um kulturelle Aspekte. Damit möchten wir die kreative Landschaft einbinden, wie z. B. Künstler, Filmmacher oder Designer. Ich denke, das bringt schon einen großen Mehrwert für das ganze Projekt und besonders für die ganzen RRI-Initiativen, weil es noch einmal einen Schritt weiter geht und die Bühne weiter öffnet. Die Methoden im akademischen Umfeld sind sehr stark auf die unmittelbare Zukunft fixiert. Anderes traut man sich zumeist nicht. Aber irgendwo müssen wir uns mit weiteren Zeithorizonten auseinandersetzen. Wenn man über Entwicklungsalternativen von Technologien sprechen will, von denen wir annehmen, dass sie weit in die Zukunft reichen, dann können wir von der künstlerischen Szene profitieren. Die Künstler, Designer und die Filmemacher haben mehr Möglichkeiten, Zukunftsthemen anzusprechen. Das ist eine Berufsgruppe, die sich nicht lächerlich macht, wenn sie über die weitere Zukunft spekuliert. Aktuelle Analysen der Gesellschaft werden imaginativ auf die Zukunft extrapoliert. Wenn man sich alte Zukunftsperspektiven anschaut, dann findet man darin oft Beschreibungen der Zustände in den damaligen Gesellschaften. Vielleicht kann man über die Kunst noch einen Schritt weitergehen, sich vielleicht aus dem Korsett der Gegenwart noch weiter befreien.

TATuP: Was sind die nächsten Pläne in Bezug auf das BIO·FICTION-Festival?

MS: Das Festival geht jetzt auf Tournee. Geplant sind über 15 Veranstaltungen auf 4 Kontinenten. Uns ist es wichtig, dass die Leute zusammenkommen und diskutieren. Diese Qualität erreicht man nicht, wenn man die Filme alleine vor dem Computer ansieht. Es geht darum, dass im Anschluss an das Filmeschauen, die gemeinsame Erfahrung und die gemeinsame Diskussion zustande kommen und die Filme als Stimulus für die Diskussion dienen.

\section{Anmerkungen}

1) $\mathrm{Zu}$ den wissenschaftlichen Beratungsgremien siehe http://ec.europa.eu/health/scientific_committees/about/; zum Gutachten zur Synthetischen Biologie siehe http://ec.europa.eu/health/scientific_committees/emerging/docs/scenihr_o_044.pdf

2) http://ec.europa.eu/health/scientific_committees/ emerging/docs/scenihr o 048.pdf

3) Das dritte Gutachten liegt noch nicht vor. Eine von der EU-Kommission initiierte Public Consultation ist vor kurzem gestartet, worauf das Gutachten aufbauen wird (http://ec.europa.eu/health/scientific_committees/consultations/public_consultations/scenihr_consultation 28 en.htm).

4) http://www.synbiosafe.eu/DVD/Synbiosafe.html

5) http://bio-fiction.com/videos/bruce-2/

\section{Weiterführende Links}

$\begin{array}{ll}\text { Festival } & \mathrm{http}: / / \text { bio-fiction.com/ } \\ \text { Videos } & \mathrm{http://bio-fiction.com/videos/} \\ \text { Biofaction } & \mathrm{http} / / \text { www.biofaction.com/ } \\ \text { Markus Schmidt } & \mathrm{http} / / \text { www.markusschmidt.eu/ } \\ \text { EU-Projekt SYNENERGENE } & \mathrm{http} / / \text { www.synenergene.eu/ }\end{array}$

$《 》$

TATuP: Vielen Dank für das Gespräch! 